\title{
Unusual Dissolution Behavior of Tooth Enamel and Synthetic HAP Crystals Under High Partial Saturation Conditions
}

\author{
M. B. FaWzI, W. I. Higuchi, and J. J. HefFErreN* \\ College of Pharmacy, University of Michigan, Ann Arbor, Michigan 48104, USA
}

The dissolution behavior of enamel and synthetic hydroxyapatite in acidic media possessing a high degree of partial saturation was found to be neither simple surface dissolution nor linear with time. Instead, a repetitive, stepwise dissolution pattern was observed. To explain this phenomenon, a model based upon a hypothesis that the crystals dissolve in a synchronized fashion was proposed.

Previous work ${ }^{1-5}$ in our laboratories had shown the utility of using physical models in investigating the mechanisms for dental enamel demineralization in acidic buffers. The approach has been to explore various physicai models for dissolution with appropriately designed experiments to determine the factors governing the rates of dissolution and their interactions on a quantitative mechanistic basis.

Several mechanisms for acid dissolution of enamel and of synthetic hydroxyapatites (HAPs) involving at least two sites have now been established.4-8 Under "sink" conditions, demineralization of enamel is apparently largely surface dissolution ${ }^{1,4-8}$ and predominantly bulk diffusion controlled (at low-tomoderate agitation conditions). Also, under sink conditions at high agitation there seems to be a significant contribution to the overall kinetics from a surface reaction step. In addition, the recent studies under partial saturation conditions have shown that the dissolution process could be largely zonal involving a region of 50 to 100 micrometers $(\mu \mathrm{m})$ thickness at the surface. The two-site model has yielded a relatively complete and unified understanding of the more complex situation of HAP dissolution under both sink

This investigation was supported by USPHS Grant DE-01830 from the National Institute of Dental Research, National Institutes of Health, Bethesda, Md.

Received for publication August 19, 1975.

Accepted for publication July 28, 1976.

* Dr. Hefferren is also associated with the Research Institute, American Dental Association Health Foundation

* Where the reference phase has a $\mathrm{K}_{\mathrm{HAP}}=10^{-115}$. ${ }^{11}$ and modest partial saturation conditions. ${ }^{9}$ In addition, a wide range of application of this new model has been developed in assessing the roles of fluoride ${ }^{10}$ and a large number of trace elements ${ }^{10}$ in apatite dissolution in acid buffers.

It is the purpose of this article to report the results of the acid demineralization behavior of dental enamel under relatively high partial saturation conditions (for example, $50 \%$ saturated with respect to $\mathrm{HAP}^{*}$ ) where a very unusual pattern of dissolution has been observed which is in striking contrast to the foregoing discussion that relates to sink conditions and to modest partial saturation conditions. These results and the similar results obtained with synthetic HAP pellets are believed to be especially important to the clinical situation involving the "white spot" formation phenomenon.

\section{Materials and Methods}

Human dental block enamel.-The human teeth, mainly ceniral incisors with sound surfaces, were provided by dentists in the Chicago area. A cylindrical plug $(4 \mathrm{~mm}$ in diameter) was cut from these teeth and prepared by mechanical polishing to provide subsurface enamel. The cut plug was mounted in a cylindrical brass ring with the enamel section on the surface using cold-curing methyl methacrylate. A one fourth-inch diameter acrylic rod was attached to the mount. The block enamel mounts were stored in double distilled water before their use in the experiment.

Preparation of synthetic HAP-Nonradioactive and radioactive synthetic $H A P$ were prepared using the procedures developed by Moreno, Gregory, and Brown. ${ }^{11}$

The radioactive HAP was prepared by the same procedure just described except radiolabeled $\mathrm{Ca}^{45}$ was used in the starting raw material. About $10 \mathrm{mCi}$ of ${ }^{45} \mathrm{CaCl}_{2}$ was used in the synthesis which yielded about $300 \mathrm{gm}$ of HAP. The specific activity was about $3.5 \cdot 10^{-2}$ 


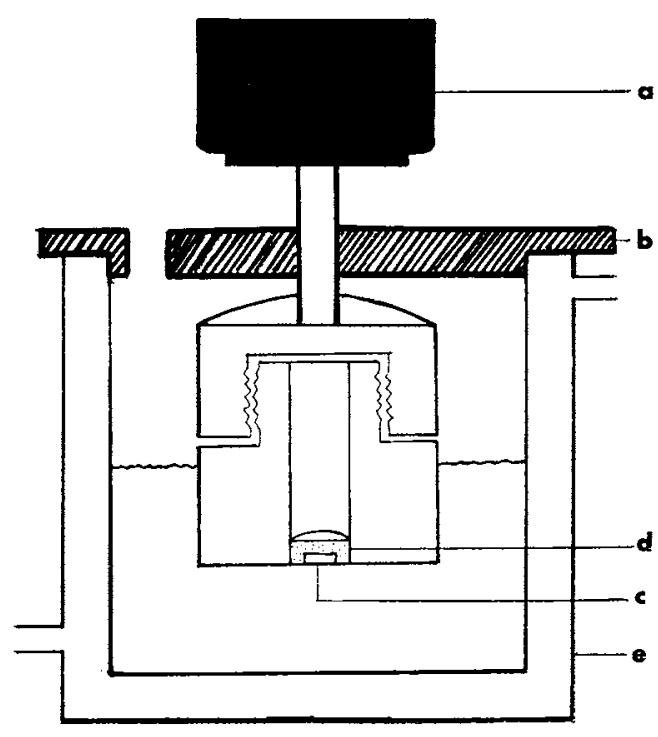

FIG 1.-Rotating disk experimental setup. $a$, constant speed motor; $b$, cover; $c$, HAP pellet; $d$, paraffin; $e$, jacketed glass beaker.

$\mathrm{mC}$ :/gm. Both products were checked by infrared and elemental analysis.

Preparation of HAP PELlets.-About $40 \mathrm{mg}$ of HAP (preequilibrated in a humidity chamber that contained saturated potassium nitrate aqueous solution to maintain humidity in the chamber at about $67 \%$ ) was directly compressed in a die $(0.62 \mathrm{~cm})$ inder a force of $10,000 \mathrm{lb}$ using a laboratory press. The resulting surface area, A, of the pellet is $0.317 \mathrm{~cm}^{2}$. The pellet is then ejected from the die and mounted on a pellet holder with melted paraffin so that only one flat surface of the HAP pellet is exposed.

Preparation of buffers.-The $0.10 \mathrm{M}$ acid buffer solutions were prepared by mixing calculated quantities of the acid and their salts. Partially saturated buffer solutions were made by adding predetermined amounts of calcium chloride or sodium dihydrogen phosphate. In both instances, calculated amounts of sodium chloride were added to maintain a constant ionic strength of 0.50 . The $\mathrm{pK}_{\mathrm{HAP}}$ of these solutions was calculated by a computer program $^{12}$ that takes into account activity coefficient corrections and ion pairs in the solution.

ANALYTICAL CHEMISTRY PROGEDURES.Methods for the analyses of phosphate and calcium used in the present work have already been described. ${ }^{13}$ Calcium was determined by

$\dagger$ Beckman Liquid Scintillation System, Beckman Instruments, Inc., Fullerton, Ca. atomic absorption spectrophotometry, and phosphate by a colorimetric procedure. ${ }^{45} \mathrm{Ca}-$ labeled HAP was anaiyzed by a liquid scintillaation counter $\dagger$

EXPERIMENTAL PROCEDURE.-HAP pellet dissolution apparatus.-Figure 1 is a schematic representation of the experimental rotating disk dissolution apparatus. The pellet was fixed in the center of the disk by melted paraffin. Before an experiment, this pellet was soaked in double distilled water overnight. This disk assembly was connected to a constant speed rotating mechanism. The motor was firmly suspended by means of clamps from a series of bars that were rigidly fastened to the laboratory bench.

The ratio of the flask diameter to disk diameter was expressed in a numerical value of 2.5 which exceeded the ratio value of 2.4 recommended by Riddiford. ${ }^{14}$ Throughout the experimental dissolution procedures, the distance of the disk from the walls and bottom of the flask exceeded $0.5 \mathrm{~cm}$, and the disk was immersed to a level that exceeded $0.5 \mathrm{~cm}^{14}$ Also, under our experimental conditions, the surfaces of both enamel and HAP pellets appeared smooth (that is, surface roughness $\sim$ $1 \mu \mathrm{m}$ ) even at the end of an experiment.

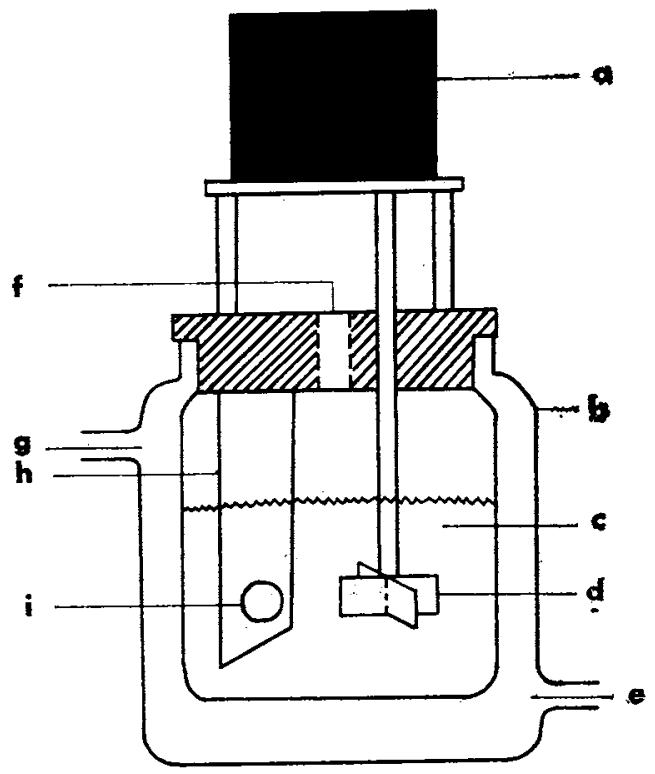

Fig 2.-Static disk dissolution apparatus. $a$, constant speed motor; $b$, jacketed glass beaker; $c$, bulk solution; $d$, stirrer; $e$, water inlet; $f$, sampling port; $g$, water outlet; $h$, pellet holder; $i$, pellet of HAP. 
Figure 2 shows a schematic representation of the experimental static disk dissolution apparatus. The pellet was mounted in the pellet holder with melted paraffin, with only one surface exposed. This surface faced the stirring paddle inserted at the top of the water-jacketed cylinder. The stirring speed was maintained at $150 \mathrm{rpm}$ in most of the experiments. Before an experiment this pellet was soaked in double distilled water overnight.

Human dental block eNamel dissoluTION APPARATUS.-The block enamel mount attached to a one fourth-inch diameter acrylic rod was connected to a constant speed rotating mechanism. 'The rest of the specifications were essentially identical to the previously mentioned rotating disk apparatus for HAP pellet (Fig 1).

The discolution rate experiments were run as follows: an acid buffer solution or partially saturated buffer $(25 \mathrm{mI})$ was accurately meas. ured and placed into the jacketed $100-\mathrm{ml}$ beaker and allowed to attain thermal equilit;rium at $30 \mathrm{C}$ (or otherwise indicated temperature of the experiment). Then the flask containing the solution was raised, using a lab jack, until the enamel surface or the pellet sur- face was immersed. At the moment of contact, the motor and a timer were simultaneously started. Then the block enamel or the pellet holder was lowered to its final position. An aliquot of sample (about $5 \mathrm{ml}$ ) was withdrawn by pipet at suitable intervals. The solution was replaced by an equal volume of the partially saturated starting buffer solution, thus maintaining a constant volume. The samples were diluted and analyzed for calcium or phosphate or both. The amount dissolved was calculated according to

$$
\begin{aligned}
& \text { mg dissolved at time } \mathrm{t}= \\
& \left(V_{\mathrm{r}} \Delta C_{\mathrm{t}}+\Sigma V_{m} \Delta C_{m}\right) \mathrm{F},
\end{aligned}
$$

where $\Delta C_{\mathrm{t}}$ is the excess (over that in the starting buffer solution) concentration at time $t$; $V_{n}$, the total volume of solvent; $V_{m}$, the volume of sample withdrawn in previous intervals; $\Delta C_{m}$, the excess concentration of the sample withdrawn in the previous interval; and $F$, a stoichiometry conversion factor depending on whether calcium or phosphate is determined.

\section{Results and Discussion}

The data obtained from dissolution experiments were plotted as the "amount of HAP dis-

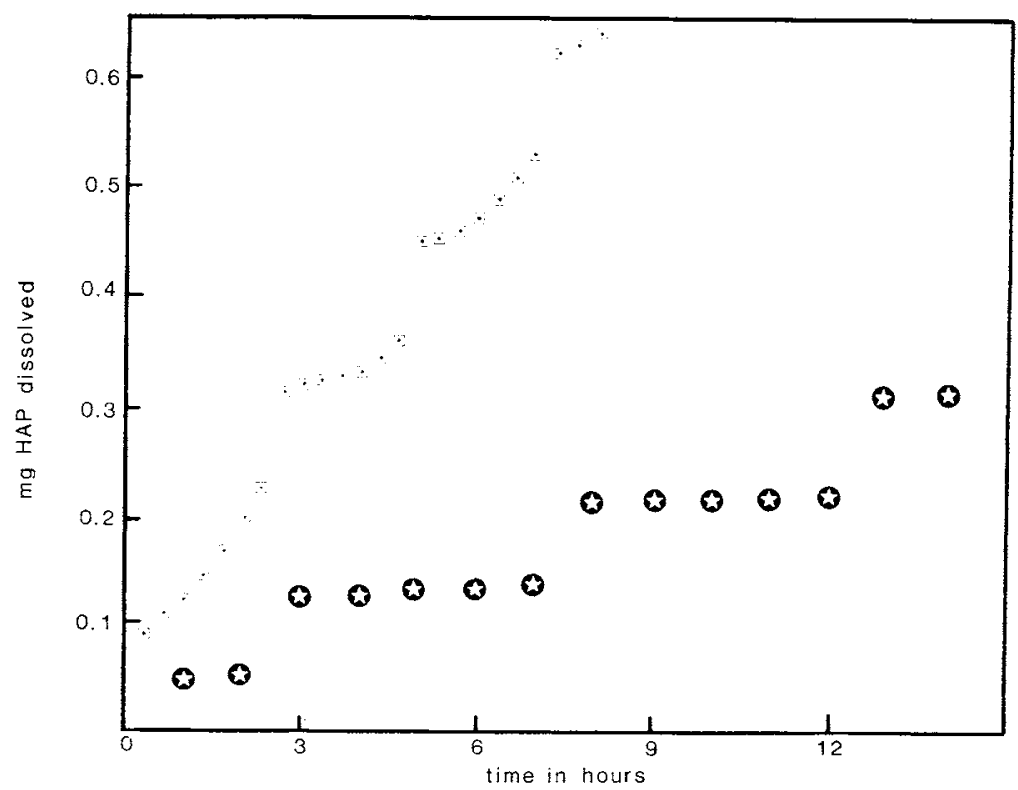

FIg 3.-Dissolution rate behavior of human dental block ( $\mathrm{A}=0.125$ $\mathrm{cm}^{2}$ ) in partially saturated acetate buffer at $500 \mathrm{rpm}$ (rotating disk). Bulk solution conditions: $0.1 M$ acetate buffer at $\mathrm{pH}$ of 4.5 and $0.5 M$ ionic strength. Open star in solid circle, $\mathrm{TCa}=4.69 \times 10^{-2} \mathrm{M} ; \mathrm{TP}=4.69 \times$ $10^{-4} \mathrm{M}$. Dotted square, TCa $=1.98 \times 10^{-2} \mathrm{M} ; \mathrm{TP}=1.98 \times 10^{-4} \mathrm{M}$. 


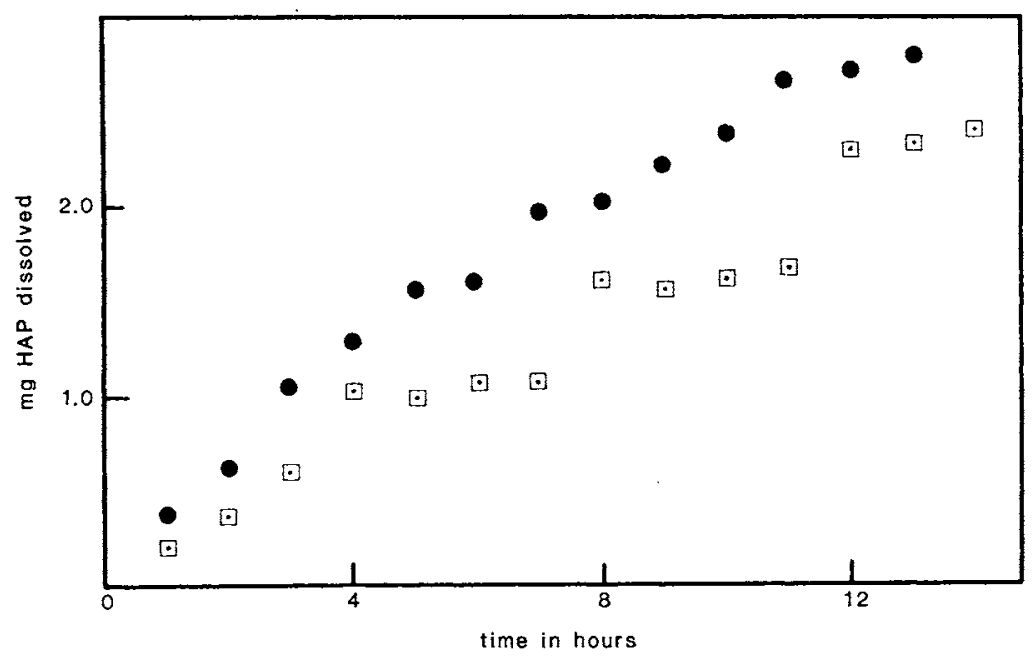

Fig 4.-Dissolution rate behavior of (NBS) HAP pellets ( $A=0.317$ $\mathrm{cm}^{2}$ ) in partially saturated acetate buffer solution at $150 \mathrm{rpm}$ (static disk). Bulk solution conditions: 0.1 acetate buffer at $\mathrm{pH}$ of $4.5 \mathrm{M}$ and $0.5 \mathrm{M}$ ionic strength. Solid circle, TCa $=1.98 \times 10^{-2} M ; \mathrm{TP}=1.98 \times 10^{-3} M$. Dotted square, $\mathrm{TCa}=8.356 \times 10^{-3} \mathrm{M} ; \mathrm{TP}=8.356 \times 10^{-4} \mathrm{M}$.

solved ( $\mathrm{mg}$ ) in $25 \mathrm{ml}$ of buffer" vs time. The amounts of HAP dissolved were calculated on the basis of both calcium and phosphate analyses whenever both were possible. Good stoichiometry, that is, congruent dissolution, was found in all experiments.

The most surprising and exciting aspect of these studies has been the unusual cyclic stepwise pattern of dissolution that occurs, especially when the partial saturation is high. Figure 3 shows some typical results for human dental block central incisor enamel dissolution in acetate buffer at a $\mathrm{pH}$ of 4.5 under two partial saturation conditions defined by $\mathrm{K}_{\mathrm{HAP}}=$ $\left(a_{\mathrm{Ca}^{2}}{ }^{2+}\right)^{10}\left(a_{\mathrm{PO}}{ }^{4}\right)^{2-6}\left(a_{\mathrm{OH}^{-}}\right)^{2}$. Note the unusual, nonlinear, stepwise dissolution. Experiments with synthetic HAP pellets have yielded similar results (Fig 4). Although the data obtained with the synthetic HAP pellets are not as clean cut as with the human dental enamel blocks, the cyclic, steplike patterns are clearly present, at least for the $\mathrm{K}_{\mathrm{HAP}}=10^{-122.5}$ experiments, and these experiments have been repeated many times. This behavior has been found under a variety of conditions and is independent of any of the following factors: (1) the calcium-phosphate ratio was varied from the numerical value expressed as 0.1 to 100 as shown in Figure 5; (2) lactate buffer (Fig 6) showed essentially the same results as acetate buffers; (3) at $37 \mathrm{C}$, the results were found to be essentially the same. as those obtained at $30 \mathrm{C}$ (Fig 7); and (4) the behavior was found to be essentially independent of the stirring rate.

These data have not yielded to any classical textbook interpretations for crystalline solid dissolution. The possibility of intermittent precipitation of new calcium phosphate phases was examined since a stepwise dissolution pattern could be associated with such a mechanism. ${ }^{15}$. However, calculations show ${ }^{12}$ that, except at the discontinuities where the slopes and therefore the rates are high, the prevailing surface solution activity products are low cnough that only HAP can exist at the enamel surface. Therefore, it would be unreasonable to implicate phases other than HAP in interpreting the "plateau" portions of these results.

The following hypothesis for the unusual dissolution behavior of HAP under partial saturation conditions has been considered. To have such stepwise patterns for dissolution, there needs to be synchronization among an assembly of crystals and there should be rather large energy or chemical potential variations at the sites of dissolution during each step. This may be related, for example, to substantial chemical potential differences among different planes in domains of the order of dimensions of the unit cell and which are in contact with the solvent. Therefore, substantial variations in the "driving force" for dissolution may occur that would be dependent on the stage of dissolution of these domains. Calculations based on known crystal 


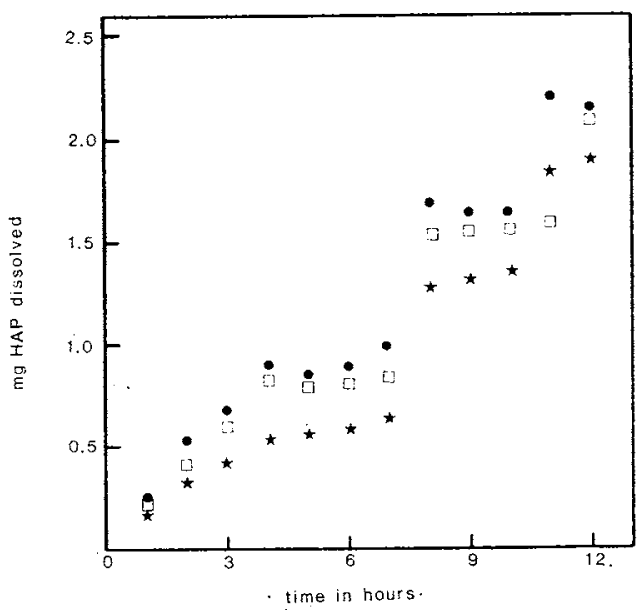

FIG 5.-Effect of TGa/TP ratio on dissolution rate behavior of (NBS) HAP pellets $\left(A=0.317 \mathrm{~cm}^{2}\right.$ ) in partially saturated acetate buffer at $500 \mathrm{rpm}$ (rotating disk). Bulk solution conditions: $0.1 M$ acetate buffer at $\mathrm{pH}$ of 4.5 and $0.5 M$ ionic strength. Solid circle, TCa $=$ $\mathrm{TP}=8.536 \times 10^{-3} M$. Open square, $\mathrm{TCa}=$ $1.98 \times 10^{-2} M ; \mathrm{TP}=1.98 \times 10^{-3} M$. Solid star, $\mathrm{TCa}=4.69 \times 10^{-2} M ; \mathrm{TP}=4.69 \times 10^{-4}$ $M$.

dimensions for the HAP crystallites are consistent with the idea that the increment of material dissolved associated with a single step (Fig 3 ) might be associated with the dissolution of domains of the order of dimension of a unit cell in planes parallel to the $\mathrm{C}$-axis.

The more important consideration is how the crystals can dissolve "in phase" which requires, for example, that crystals with surface domains in some stage of dissolution may be slowed up while those that are "lagging" are permitted to catch up. Synchronization may be achieved in the following manner. The ionic activity product in the aqueous phase of the enamel would be high following a "burst" of dissolution (Fig 3). This high ambient solution concentration of ions would then retard or inhibit dissolution from crystals having domains in such stages of dissolution that the surface ion activity products would be lower than that in the ambient fluids. If the lagging crystals have higher surface ion activity products (or "solubility"), these would dissolve and catch up to those that have been retarded and thus all of the crystals become in phase. Now the ion concentrations in the aqueous phase

* Since the submission of this article, we were informed by $G$. H. Nancollas that he has been able to observe these periodic, stepwise dissolutions in lactate and acetate buffers. (pores) of the enamel slowly decrease as a result of the diffusion of these ions out of enamel and into the external solution. When the ambient solution ion concentration in the enamel again reaches a sufficiently low value (corresponding to the lowest energy plane in a dissolving domain) as a result of this diffusional relaxation process, sudden dissolution of all of the synchronized crystals will take place. This process will repcat itself until the assembly has completely dissolved.

To test this synchronized dissolution hypothesis, an experiment has been conceived that involves dialyzing a low slurry density HAP suspension in a well-stirred cellophane dialysis bag and determining the concentrations inside and outside the bag as a function of time. The results of these experiments and their detailed analysis are given in an earlier article in this Journal. ${ }^{16}$ Periodic oscillations of the ion concentrations in the bag have been observed and these findings strongly support the synchronized dissolution hypothesis and the theory that the HAP crystals in dental enamel may be dissolving in phase, so to speak, bursting forth ions periodically with a diffusional relaxation period in between.

\section{Conclusions}

We believe that these findings* are of significant importance to the understanding of the

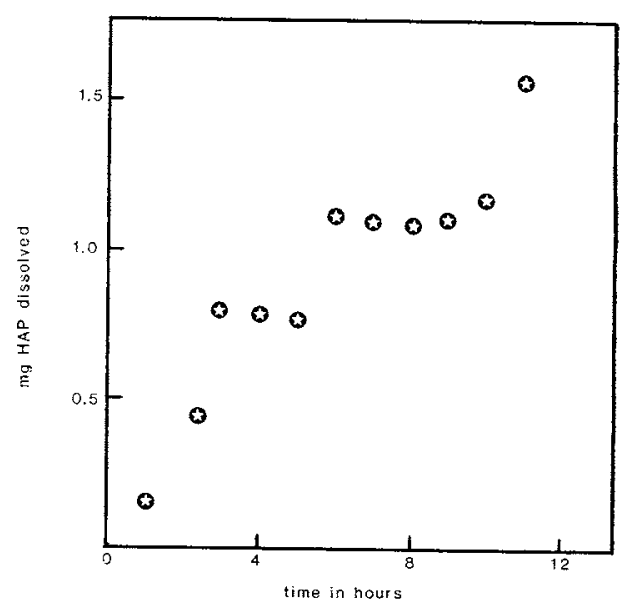

Fig 6.-Dissolution rate behavior of human dental enamel block $\left(A=0.129 \mathrm{~cm}^{2}\right)$ in partially saturated lactate buffer at 500 rpm (rotating disk). Bulk solution conditions: $0.1 M$ lactate buffer at $\mathrm{pH}$ of $4.5 \mathrm{M}$ ionic strength. $\mathrm{TCa}=4.69 \times 10^{-2} M ; \mathrm{TP}=4.69 \times 10^{-4} M$. 


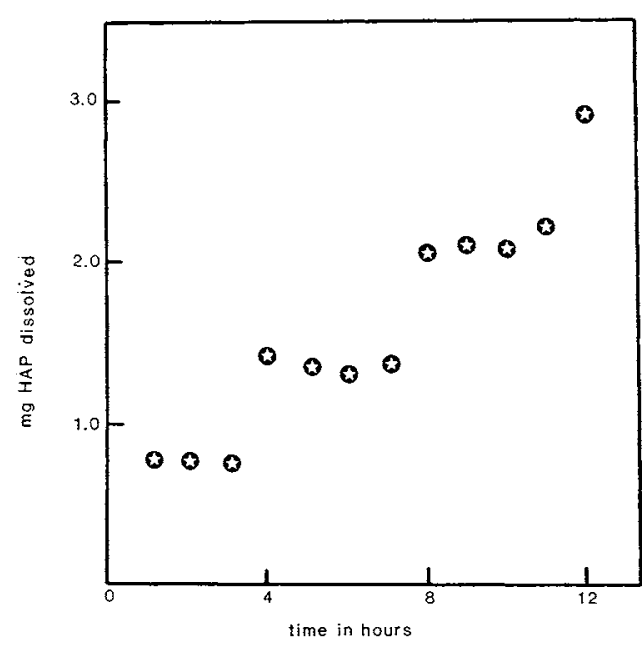

FIg 7.-Dissolution rate behavior of (NBS) HAP pellet $\left(A=0.317 \mathrm{~cm}^{2}\right)$ in partially saturated acetate buffer at $37 \mathrm{C}$ and $150 \mathrm{rpm}$ (static disk). Bulk solution conditions: $0.1 \mathrm{M}$ acetate buffer at $\mathrm{pH}$ of 4.5 and $0.5 M$ ionic strength. $\mathrm{TCa}=4.69 \times 10^{-2} M ; \mathrm{TP}=4.69 \times$ $10^{-4} \mathrm{M}$.

mechanism of dental caries and the understanding of the mechanism of action of various agents on the relevant demineralization process. It is likely that during in vivo dental caries formation a moderate dental "plaque attack" should result in a moderate partial undersaturation at the tooth-plaque interface rather than an undersaturation approaching sink conditions. Thus, the mechanism described in this report is more likely to be the mechanism governing demineralization during in vivo dental caries rather than the sink dissolution mechanism. Furthermore, it is well acknowledged that white spot formation (subsurface demineralization) is a first step in dental caries and this is consistent with the zonal dissolution feature of the proposed mechanism.

\section{References}

1. Higuchi, W.I.; Gray, J.A.; Hefferren, J.J.; and PATEL, P.R.: Mechanisms of Enamel Dissolution in Acid Buffers, $J$ Dent Res 44:330-341, 1965.

2. Higuchl, W.I.; Mtr, N.A.; PAtel, P.R.; Becker, J.W.; and Hefferren, J.J.: Quantitation of Enamel Demineralization Mechanisms: III. A Critical Examination of the Hydroxyapatite Model, J Dent Res 48:396$409,1969$.

3. Mrr, N.A., and Higuari, W.I.: The Mechanism of Action of Solution Fluoride upon the Demineralization Rate of Human
Enamel, Arch Oral Biol 14:901-920, 1969.

4. Young, F.; Fawzi, M.B.; DedhiYa, M.G.; Wu, M.S.; and Higuahr, W.I.: Dual Mechanisms for Dental Enamel Dissolution in Acid Buffers, J Dent Res 53 (Special Issue): Abstract No. 57, 1974.

5. Fox, J.L.; FawzI, M.B.; Higuchi, W.I.; HefFerReN, J.J.; and HwU, R.G.: A TwoSite Model for Enamel Dissolution in EDTA, $J$ Dent Res 53 (Special Issue): Abstract No. 586, 1974.

6. Higuchi, W.T., in Rowe, N.A. (ed): Dental Plaque: Interface, Ann Arbor, Mich: University of Michigan Press, 1973, pp 74-78.

7. Wu, M.S., and HIGUCHI, W.I.: Dissolution Rate Studies with Synthetic Hydroxyapatite Pellets in Acidic Buffers Employing the Rotating Disk Method, J Dent Res 55:496505, 1976 .

8. Fawzi, M.B.; Young, F.; Higuchi, W.I.; and Hefrerren, J.J.: Unusual Dissolution Behavior of Enamel in Partially Saturated Acidic Media, J Dent Res 53 (Special Issue): Abstract No. 868, 1974.

9. Fox, J.L.; FAwzI, M.B.; WU, M.S.; and Higuchi, W.I.: A New Two-Site Model for Hydroxyapatite Dissolution Acidic Media, Arch Oral Biol, submitted for publication.

10. Wu, M.S.; HigughI, W.I.; DedhiYa, M.G.; Shimabayashi, S.; and Hefferren, J.J.: Rotating Disk Method Studies of Dental Enamel and Synthetic Hydroxyapatite Dissolution, J Dent Res 54 (Special Issue A): Abstract No. L538, 1975.

11. Moreno, E.C.; Gregory, T.M.; and Brown, W.E.: Preparation and Solubility of Hydroxyapatite, $J$ Research, NBS, 72A: $773,1968$.

12. Fox, J.L., and Higuchi, W.I.: Methodologies for Transport Calculations Involving Diffusion, Complex Chemical Equilibria, and Crystal Kinetics, J Dent Res, submitted for publication.

13. AMin, K.N.: Solubility of Hydroxyapatite and Its Inhibition by Phosphate, Ph.D. thesis, University of Michigan, 1971.

14. Riddiford, A.C.: The Rotating Disk System, in Delahay, P. (ed): Advances in Electrochemistry and Electrochemical Engineering, Vol 4, New York: Interscience, $1966, \mathrm{p} 47$.

15. Higughi, W.I.; Mir, N.A.; Parker, A.P.; and Hamlin, W.E.: Dissolution Kinetics of a Weak Acid, 1,1-Hexamethylene $p$-Tolysulfonylsemicarbazide, and Its Sodium Salt, $J$ Pharm Sci 54:8-11, 1965.

16. FaWzi, M.B.; Sonobe, T.; Higuahi, W.I.; and Hefrerren, J.J.: Synchronized Crystal Dissolution Behavior for Tooth Enamel and Synthetic (NBS) HAP, $J$ Dent Res 56:394$406,1977$. 